\title{
A respiração frenolabial na doença pulmonar obstrutiva crônica: revisão da literatura
}

\author{
Pursed-lip breathing in chronic obstructive pulmonary disease: a literature review \\ Renata Claudino Rossi', Carlos Marcelo Pastre², Ercy Mara Cípulo Ramos², \\ Luiz Carlos Marques Vanderlei ${ }^{2}$
}

\begin{abstract}
RESUMO I Esta revisão teve por objetivo destacar os principais achados publicados nos últimos dez anos sobre os efeitos da respiração frenolabial (RFL) em pacientes com doença pulmonar obstrutiva crônica (DPOC). A busca dos artigos foi realizada nas bases de dados Lilacs, IBECS, MEDLINE e SciELO, por meio dos seguintes descritores da área da saúde (DeCS): doença pulmonar obstrutiva crônica, reabilitação, respiração, hiperinsuflação e dispneia, e suas respectivas versões na língua inglesa (MeSH), além do termo pursed-lip breathing. Após a eliminação dos títulos repetidos, foram selecionados somente os estudos que abordavam a RFL como tema principal, resultando em 12 artigos científicos, 10 ensaios clínicos e 2 revisões bibliográficas. Segundo os achados, a RFL proporciona: alterações sobre a gasometria arterial, caracterizada pelo aumento da saturação e pressão parcial de oxigênio; padrão ventilatório, com diminuição da frequência respiratória e aumento de tempo expiratório e do volume corrente; mecânica ventilatória, por meio do recrutamento de musculatura abdominal expiratória e dos músculos da caixa torácica e acessórios da inspiração; diminuição no consumo de oxigênio; alterações na modulação autonômica cardíaca induzida pelo aumento da atividade parassimpática e, por fim, melhora na qualidade de vida destes pacientes. A RFL é considerada uma manobra de grande importância, por repercutir de forma positiva em diversos sistemas e sobre a qualidade de vida de pacientes portadores da DPOC.
\end{abstract}

Descritores | doença pulmonar obstrutiva crônica; dispneia; respiração.
ABSTRACT I This review aimed at standing out the main findings published in the last ten years on the effects of pursed-lip breathing (PLB) in patients with chronic obstructive pulmonary disease (COPD). The search for articles was performed in Lilacs, IBECS, MEDLINE, and SciELO database by using the descriptors of health (DeCS): doença pulmonar obstrutiva crônica, reabilitação, respiração, hiperinsuflação e dispneia, and their respective versions in the English language (MeSH), the term pursed-lip breathing was also in such list. After removing the repeated titles, only studies addressing PLB as the main theme were selected, resulting in 12 papers, 10 clinical trials, and 2 systematic reviews. According to the findings, the PLB provides: changes on arterial gases, which are characterized by increased oxygen saturation and partial pressure of oxygen; ventilatory pattern with decreased respiratory rate and increased expiratory time and tidal volume; respiratory mechanics, by recruiting the expiratory abdominal muscles and muscles of the rib cage and accessories inspired; decrease in oxygen consumption; alterations in cardiac autonomic modulation induced by increase in parasympathetic activity and, ultimately, improved quality of life of these patients. The PLB is considered a maneuver of great importance to have a positive effect on various systems and on the quality of life of patients with COPD.

Keywords | chronic obstructive pulmonary disease; dyspnea; respiration. 


\section{INTRODUÇÃO}

Defendida desde 1910 por Saenger como uma forma de exercício físico ${ }^{1}$, a respiração frenolabial (RFL) é caracterizada como uma manobra ventilatória, baseada em uma inspiração nasal seguida de uma expiração resistida, executada por meio dos lábios franzidos e/ou dentes semicerrados, com relação tempo inspiratório e tempo expiratório (TI:TE) de 1:32. Por produzir um padrão respiratório mais fisiológico e eficiente, a RFL é amplamente utilizada por diversos tipos de populações, tais como indivíduos saudáveis ${ }^{3}$, portadores de distrofia muscular ${ }^{4}$ e por pacientes com doença pulmonar obstrutiva crônica (DPOC), sob forma espontânea ou em programas de reabilitação pulmonar ${ }^{5-9}$.

A manobra desencadeia alterações, caracterizadas pelo aumento dos níveis de oxigênio arteriais $\left(\mathrm{PaO}_{2}\right)$ e saturação arterial de oxigênio $\left(\mathrm{SpO}_{2}\right)^{4,7}$, bem como diminuição da taxa do dióxido de carbono $\left(\mathrm{CO}_{2}\right)^{5}$, decorrentes do padrão ventilatório específico proporcionado pela RFL, ou seja, diminuição da frequência respiratória $(f)$ e aumento do volume corrente (VC), o que pode ou não alterar o volume minuto ${ }^{6,7}$.

Além disso, a RFL mantém pressão positiva nas vias aéreas acrescida da diminuição da velocidade do fluxo expiratório que atua diretamente na diminuição do efeito de Bernoulli, o que evita a tendência de seu colapso ${ }^{5,10}$. No âmbito da mecânica ventilatória, verifica-se uma alteração no recrutamento da musculatura respiratória, marcada pelo aumento da atuação da musculatura expiratória abdominal, dos músculos da caixa torácica e acessórios da inspiração bem como uma menor atuação do diafragma, prevenindo a fadiga muscular respiratória precoce ${ }^{3,8}$.

Ademais, a execução da manobra por pacientes com DPOC pode estar associada à melhora da qualidade de vida, devido ao fato de atuar no quadro de ansiedade e pânico, desencadeado pela presença intensa dos sintomas da doença ${ }^{7,8}$.

Diante do impacto positivo que a RFL exerce tanto no tratamento fisioterapêutico de paciente com DPOC, em programas de reabilitação pulmonar, quanto nas atividades de vida diária e qualidade de vida deste tipo de população, entende-se a importância do estudo em reunir informações sobre o tema. Para isso, a presente revisão teve por finalidade a atualização dos recentes achados publicados nos últimos dez anos que abordaram os efeitos da RFL em pacientes com DPOC.

\section{METODOLOGIA}

\section{Estratégia de busca}

A busca dos artigos selecionados para este estudo foi realizada em dezembro de 2010 e utilizou as bases de dados Lilacs, IBECS, MEDLINE e SciELO. Para esta pesquisa foram feitos os cruzamentos dos seguintes descritores da área da saúde (DeCS): doença pulmonar obstrutiva crônica, reabilitação, respiração, hiperinsuflação e dispneia, e suas respectivas versões na língua inglesa (MeSH). Adicionalmente, foi utilizado o termo pursed-lip breathing (RFL), que, mesmo não sendo considerado um descritor pelo DeCS, foi incluso devido à sua larga utilização como palavra-chave.

Para esta revisão, inicialmente, realizou-se a triagem dos títulos relacionados ao tema em questão, ou seja, que abordassem como ideia principal a reabilitação da DPOC; o treinamento respiratório e técnicas ou padrões respiratórios utilizados na prática clínica; títulos que apresentassem o termo RFL ou alguma informação referente a tal palavra-chave como pressão positiva nas vias aéreas e respiração com resistência; e, por fim, aqueles que abordassem atuações sobre o quadro de dispneia e hiperinsuflação na DPOC. Ao final da busca, foram excluídos os repetidos, já que a presente pesquisa foi realizada em diversas bases de dados.

Em seguida, foi feita a leitura detalhada dos resumos dos artigos a fim de selecionar aqueles que abordassem exclusivamente os efeitos da RFL em pacientes portadores de DPOC. Excluídos os resumos que não versavam sobre o tema, os textos completos foram avaliados e os que não se engajavam no fator de exclusão foram inclusos como resultado final da busca. Além disso, as referências dos estudos selecionados foram revisadas a fim de complementar a pesquisa. Todas as etapas da busca foram realizadas por somente um avaliador, com a supervisão de um revisor sênior.

\section{Critérios de inclusão}

Como critérios de inclusão, foram selecionados somente estudos publicados entre o período de 2001 a 2010 nos idiomas inglês, português e espanhol. Devido à escassez de artigos que abordam o tema, não foram feitas restrições quanto ao desenho do estudo. 


\section{Critério de exclusão}

Foram excluídos da busca os achados publicados como editoriais.

\section{Análise dos dados}

Os dados foram descritos de forma qualitativa e tabulados de acordo com os autores e o ano do estudo, a descrição da população, o protocolo realizado, as variáveis estudadas e os resultados. Para os artigos selecionados, que não possuíam sua versão completa disponível, utilizou-se como base seu respectivo resumo. As revisões bibliográficas foram apresentadas de forma descritiva por não possuírem o padrão de tabulação estipulado pelo presente estudo.

\section{RESULTADOS}

A estratégia utilizada na busca e os resultados podem ser visualizados na Figura 1, organizados como um fluxograma para um melhor entendimento das etapas realizadas no processo de seleção dos artigos.

\section{Características dos estudos selecionados}

Os Quadros 1 e 2 descrevem os dez ensaios clínicos selecionados ${ }^{11-20}$. As duas revisões bibliográficas encontradas na busca realizada estão apresentadas a seguir de forma descritiva, por não possuírem a formatação estabelecida para a análise dos dados.

A primeira revisão da literatura selecionada é de autoria de Fregonezzi et al. ${ }^{21}$, publicada em 2004. A revisão aborda o impacto da RFL sobre variáveis como função pulmonar, gases arteriais, padrão respiratório, músculos respiratórios e sua implicação clínica. Segundo os autores, a manobra pode ser utilizada em programas de reabilitação respiratória como estratégia para aprimorar o padrão ventilatório de pacientes portadores de DPOC, bem como de asma e doenças que afetam o sistema respiratório ${ }^{21}$.

Também em 2004, Dechman et al. ${ }^{22}$ publicaram outra revisão que abordou a RFL e a respiração diafragmática como padrões de treinamento respiratório para DPOC. Em relação à RFL, foram relatados seus efeitos sobre ventilação e função pulmonar, dispneia, trabalho respiratório, consumo de oxigênio e suas implicações clínicas. A revisão reforça o benefício da realização da

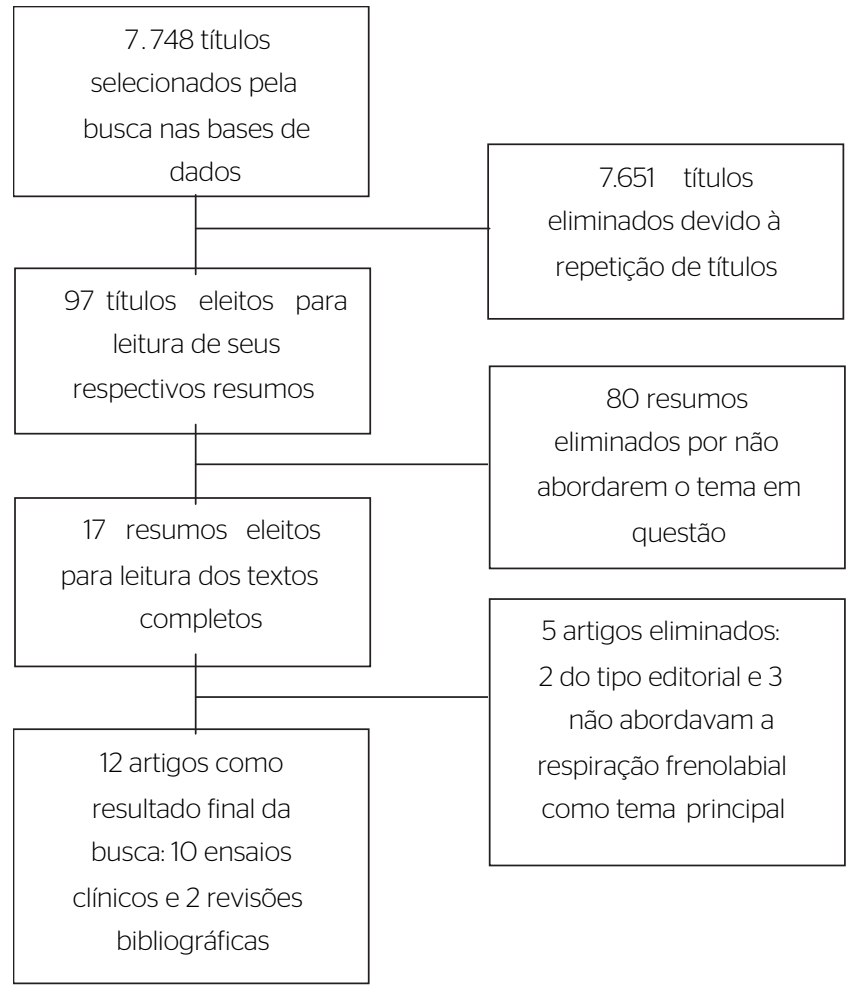

Figura 1. Fluxograma das informações sobre as diferentes etapas da busca

técnica no alívio de sintomas dos pacientes, bem como os prejuízos apresentados por sua execução por aqueles que não a adotam espontaneamente, evidenciando o cuidado em sua utilização ${ }^{22}$.

\section{DISCUSSÃO}

Os ensaios clínicos selecionados abordaram os efeitos da RFL sobre a mecânica ventilatória, o padrão respiratório, o consumo de oxigênio e a modulação autonômica cardíaca em pacientes com DPOC. Para uma melhor compreensão dos resultados, estes tópicos serão abordados separadamente.

\section{Respiração frenolabial e mecânica respiratória}

Pacientes com DPOC possuem alteração na dinâmica muscular respiratória decorrente da hiperinsuflação, caracterizada por uma desvantagem mecânica ocasionada pelo aplainamento do diafragma, que se apresenta encurtado com menor zona de aposição, a qual repercute, consequentemente, na diminuição do potencial de geração de força ${ }^{23}$. 
Quadro 1. Descrição dos principais achados dos efeitos da respiração frenolabial sobre a doença pulmonar obstrutiva crônica

\begin{tabular}{|c|c|c|c|c|}
\hline Autor/Ano do estudo & População & Protocolo & Variáveis estudadas & Resultados \\
\hline $\begin{array}{l}\text { Jones A.Y.M. } \\
\text { et al., 2003"1 }\end{array}$ & $\begin{array}{l}30 \text { sujeitos } \\
\text { (68,5 } \pm 7,83 \text { anos), } \\
\text { VEF }_{1}(\% \text { predito): } \\
39,0 \pm 12,53\end{array}$ & $\begin{array}{l}\text { Consumo de } \mathrm{O}_{2} \text { mensurado } \\
\text { pela análise de gases expirados, } \\
\text { variáveis em } \mathrm{RE} \text { captadas após } \\
\text { repouso de } 30 \text { minutos e durante } \\
\text { exercícios respiratórios analisados } \\
\text { em } 10 \text { minutos, de forma aleatória. }\end{array}$ & $\mathrm{VO}_{2}, f, \mathrm{FC}$ e $\mathrm{SpO}_{2}$ & $\begin{array}{l}\text { FC estável e } \mathrm{SpO}_{2} \text { acima de } 90 \% \text { durante } \\
\text { todo protocolo, } f \text { e consumo de } \mathrm{O}_{2} \text { menores } \\
\text { durante a RFL quando comparada à RE } \\
(12,8 \pm 3,53 \text { versus } 17,3 \pm 4,23) \text { e }(164,8 \pm 20,9 \\
\text { versus } 174,5 \pm 25,2) \text {, respectivamente. }\end{array}$ \\
\hline $\begin{array}{l}\text { Bianchi R. } \\
\text { et al., 2004'2 }\end{array}$ & $\begin{array}{l}22 \text { sujeitos }(71 \pm 7 \\
\text { anos), } V F_{1} \\
\text { (\% predito): } 43 \pm 16\end{array}$ & $\begin{array}{l}\text { Volume da caixa torácica e de } \\
\text { seus compartimentos mensurados } \\
\text { em repouso, por meio da } \\
\text { pletismografia optoeletrônica, } \\
\text { durante } 6 \text { minutos de RE e } \\
6 \text { minutos de RFL. Dispneia } \\
\text { mensurada antes e após a RFL. }\end{array}$ & $\begin{array}{l}\text { Padrão ventilatório, } \\
\text { dispneia e volumes } \\
\text { da caixa torácica }\end{array}$ & 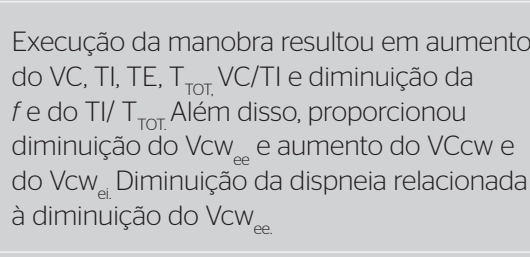 \\
\hline $\begin{array}{l}\text { Garrod R. } \\
\text { et al., } 2005^{13}\end{array}$ & $\begin{array}{l}69 \text { sujeitos, } 68 \\
\text { (51-83) anos, } \\
\operatorname{VEF}_{1}(\% \text { predito): } \\
\quad 44,3 \pm 18,8\end{array}$ & $\begin{array}{l}\text { Protocolo constituído de dois ISWT, } \\
\text { seguido de seu respectivo período } \\
\text { de recuperação, com e sem a RFL. }\end{array}$ & Dispneia, $f$, FC e $\mathrm{SpO}_{2}$ & $\begin{array}{l}\text { Não houve diferença na distância percorrida } \\
\text { e dispneia entre os ISWT. A manobra inferiu } \\
\text { em valores inferiores da } f \text { ao final do teste, } \\
\text { comparados à RE, bem como menor } \\
\text { aumento da } f \text { durante o teste. Além disso, } \\
\text { houve melhor recuperação da dispneia } \\
\text { quando realizada com RFL. Pacientes com } \\
\text { respostas positivas à RFL apresentaram } \\
\text { maior dispneia basal. }\end{array}$ \\
\hline $\begin{array}{l}\text { Spahija J. } \\
\text { et al., 2005'14 }\end{array}$ & $\begin{array}{l}8 \text { sujeitos }(58 \pm 11 \\
\text { anos), } V E F_{1}(\% \\
\text { predito): } 50 \pm 21\end{array}$ & $\begin{array}{l}\text { Carga do ergômetro, bicicleta } \\
\text { ergométrica, estipulada por um } \\
\text { teste incremental. Protocolo } \\
\text { estipulado por } 8 \text { minutos de RE e } \\
8 \text { minutos RFL, durante o repouso } \\
\text { e da mesma forma em exercício } \\
\text { (60\% da carga alcançada no teste } \\
\text { incremental). }\end{array}$ & $\begin{array}{l}\text { Dispneia, mecânica } \\
\text { ventilatória, padrão } \\
\text { ventilatório e EELV }\end{array}$ & $\begin{array}{l}\text { Efeito conflitante sobre a dispneia durante } \\
\text { exercício, sendo aumentada em quatro } \\
\text { pacientes, inalterada em dois e diminuída } \\
\text { em dois. Alterações nos escores de dispneia } \\
\text { correlacionados a mudanças no EELV. } \\
\text { RFL, em repouso e em exercício, prolonga } \\
\text { duração da respiração, diminui a fe } \\
\text { aumenta o VC. Aumento do recrutamento } \\
\text { da musculatura abdominal em repouso e } \\
\text { mais acentuado durante o exercício em três } \\
\text { pacientes com a execução da RFL. Aumento } \\
\text { da razão Ppl/Pcapl com a manobra, } \\
\text { observado somente no repouso. }\end{array}$ \\
\hline $\begin{array}{l}\text { Nield M.A. } \\
\text { et al., 200715 }\end{array}$ & $\begin{array}{c}40 \text { sujeitos }(65 \pm 9 \\
\text { anos), } V E F_{1}(\% \\
\text { predito): } 39 \pm 13\end{array}$ & $\begin{array}{l}\text { Pacientes selecionados a partir } \\
\text { de uma triagem (Borg } 3 \text { ou } \\
\text { maior no TC6'). Seleção para } \\
3 \text { tipos de treinamento, RFL, } \\
\text { EMT e controle, de } 12 \text { semanas } \\
\text { de forma randomizada com } \\
\text { acompanhamentos semanais e } \\
\text { reavaliações nas } 4^{a} \text { e } 12^{a} \text { semanas } \\
\text { de treinamento. }\end{array}$ & $\begin{array}{l}\text { Dispneia e } \\
\text { performance } \\
\text { funcional }\end{array}$ & $\begin{array}{l}\text { Melhora significativa na pontuação da } \\
\text { escala de Borg e nos valores da } \mathrm{PI}_{\text {máx }} \text { foram } \\
\text { observados apenas com a realização da RFL } \\
\text { após } 12 \text { semanas de treinamento em relação } \\
\text { ao basal, bem como valores menores } \\
\text { de } \mathrm{SpO}_{2} \text { nos TC6' realizados nas } 4^{\mathrm{a}} \text { e } 12^{\mathrm{a}} \\
\text { semanas; a manobra também proporcionou } \\
\text { melhora na capacidade funcional } \\
\text { mensurada pelo questionário SF-36. }\end{array}$ \\
\hline
\end{tabular}

$V E F_{1}$ volume expiratório forçado no primeiro minuto; RE: respiração espontânea; $\mathrm{VO}_{2}$ : consumo de oxigênio; f: frequência respiratória; FC: frequência cardíaca; SpO RFL: respiração frenolabial; EMT: treinamento de músculos expiratórios; VC: volume corrente; TI: tempo inspiratório; TE: tempo expiratório; $T$ · tempo total: Vcw : volume expiratório final da caixa torácica; VCcw: volume corrente da caixa torácica; VCW: volume inspiratório final da caixa torácica; ISWT: incremental shuttle walking test; EELV: volume pulmonar expiratório final; PpI/Pcapl: pressão pleural/capacidade de geração de pressão inspiratória estática máxima; TC6': teste de caminhada de seis minutos; PI

A realização da RFL impacta positivamente na mecânica pulmonar de pacientes com DPOC, como verificado em achados recentes da literatura. Bianchi et al. ${ }^{12}$ estudaram, em repouso, os volumes e as variações dos compartimentos da caixa torácica durante a RFL por meio da pletismografia optoeletrônica. Os resultados demonstraram que a manobra promoveu um decréscimo do volume expiratório final da caixa torácica, principalmente do compartimento abdominal, decorrente do alongamento do tempo expiratório (TE) e do tempo total do ciclo respiratório $\left(\mathrm{T}_{\mathrm{TOT}}\right)$. Além disso, o aumento do $\mathrm{T}_{\mathrm{TOT}}$ por meio do incremento em tempo inspiratório (TI) e TE, refletiu na elevação do $\mathrm{VC}$ da caixa torácica, com maior participação do VC do compartimento costal. Em relação ao volume inspiratório final da caixa torácica, a participação costal também se torna mais relevante, contudo a ação abdominal atua de certa forma por explorar o volume de reserva expiratório, o que limita o volume inspiratório final de atingir a capacidade pulmonar total. Por fim, os autores associaram a diminuição da sensação de dispneia com o decréscimo no volume da caixa torácica, sendo seu volume expiratório final eleito como preditor em relação à 
Quadro 2. Descrição dos principais achados dos efeitos da respiração frenolabial sobre a doença pulmonar obstrutiva crônica

\begin{tabular}{|c|c|c|c|c|}
\hline Autor/Ano do estudo & População & Protocolo & Variáveis estudadas & Resultados \\
\hline $\begin{array}{l}\text { Bianchi R. } \\
\text { et al., } 2007^{16}\end{array}$ & $\begin{array}{l}30 \text { sujeitos }(71 \pm 7 \\
\text { anos), } V E F_{1} \\
\text { (\% predito): } 45 \pm 16\end{array}$ & $\begin{array}{l}\text { Volume da caixa torácica } \\
\text { e de seus compartimentos } \\
\text { mensurados em repouso, } \\
\text { por meio da pletismografia } \\
\text { optoeletrônica, durante } 6 \\
\text { minutos de RE e } 6 \text { minutos de } \\
\text { RFL. Dispneia mensurada antes } \\
\text { e após a RFL. }\end{array}$ & $\begin{array}{c}\text { Dispneia, volumes da } \\
\text { caixa torácica e padrão } \\
\text { ventilatório }\end{array}$ & 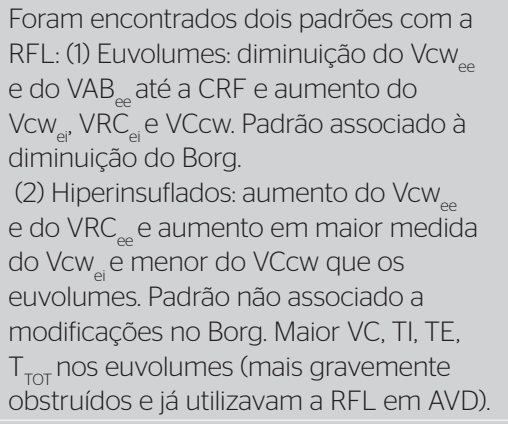 \\
\hline $\begin{array}{l}\text { Faager G. } \\
\text { et al., 2008 }\end{array}$ & $\begin{array}{l}32 \text { sujeitos, GOLD } \\
\text { II: } 69 \pm 4 \text { anos, VEF } \\
\text { (\% predito): } 61 \pm 9 ; \\
\text { GOLD III: } 65 \pm 5 \text { anos, } \\
\text { VEF }(\% \text { predito): } 37 \pm 5 ; \\
\text { GOLD IV: } 65 \pm 6 \text { anos, } \\
\text { VEF }_{1}(\% \text { predito): } 22 \pm 5\end{array}$ & $\begin{array}{l}2 \text { ESWT realizados com RFL } \\
\text { e sem RFL. Variáveis como } \\
\mathrm{SpO}_{2} \text {, FC, dispneia e fadiga } \\
\text { em membros inferiores e } \\
\text { pico de fluxo expiratório } \\
\text { foram mensurados no início, } \\
\text { imediatamente após o ESWT, } \\
\text { cinco e dez minutos após o } \\
\text { teste. }\end{array}$ & $\begin{array}{l}\mathrm{SpO}_{2}, \mathrm{FC} \text {, dispneia, } \\
\text { fadiga dos membros } \\
\text { inferiores, pico de fluxo } \\
\text { expiratório e tempo de } \\
\text { caminhada }\end{array}$ & $\begin{array}{l}\text { O ESWT realizado com a RFL resultou em } \\
37 \text { segundos a mais de caminhada do } \\
\text { que com a RE. } \mathrm{SpO}_{2} \text { diminuiu em ambos } \\
\text { os testes, porém com queda menor com } \\
\text { a RFL. Sem diferença entre os testes em } \\
\text { todas as outras variáveis estudadas. }\end{array}$ \\
\hline $\begin{array}{l}\text { Ramos E.M.C. } \\
\text { et al., } 2009^{18}\end{array}$ & $\begin{array}{c}16 \text { sujeitos }(64 \pm 11 \\
\text { anos), } V E F_{1} \\
\text { (\% predito): } 60 \pm 25\end{array}$ & $\begin{array}{l}\text { VFC analisada durante o } \\
\text { repouso em } 3 \text { etapas: } 10 \text { minutos } \\
\text { de RE, } 8 \text { minutos de RFL e } 10 \\
\text { minutos de RE. Variáveis } f, \mathrm{SpO}_{2} \\
\text { e PA foram mensuradas no final } \\
\text { de cada etapa. }\end{array}$ & $\begin{array}{c}f, \mathrm{SpO}_{2}, \mathrm{FC}, \mathrm{PA}, \mathrm{RMSSD}, \\
\Delta \mathrm{l}-\mathrm{E} \text { e } \Delta \mathrm{R}-\mathrm{RFL}\end{array}$ & $\begin{array}{l}\text { A realização da manobra resultou na } \\
\text { diminuição da } f \text {, no aumento da } \mathrm{SpO}_{2} \text { e } \\
\text { do índice RMSSD, quando comparado à } \\
\text { RE. O } \Delta \text { I-E foi 8,98 e } \Delta \text { R-RFL foi } 8,25 \text { com } \\
\text { utilização da RFL. }\end{array}$ \\
\hline $\begin{array}{l}\text { Spahija J. } \\
\text { et al., 201019 }\end{array}$ & $\begin{array}{l}57 \text { sujeitos, } V_{1} F_{1} \\
\text { (\% predito): } 44,3 \pm 17,4\end{array}$ & $\begin{array}{l}\text { Teste incremental realizado em } \\
\text { bicicleta ergométrica. Variáveis } \\
\text { estudadas mensuradas em } \\
\text { ambos os testes. }\end{array}$ & $\begin{array}{l}\text { Wpeak, } \mathrm{VO}_{2} \text { pico, } \\
\text { padrão ventilatório e } \\
\text { dispneia }\end{array}$ & 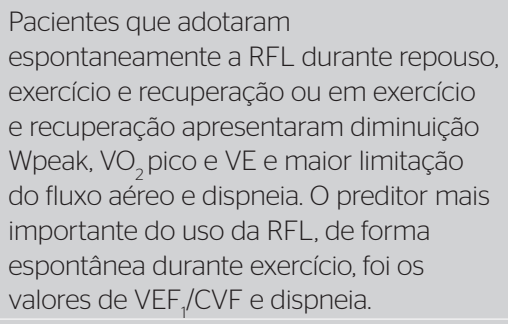 \\
\hline $\begin{array}{l}\text { Visser F.J. } \\
\text { et al., } 2011^{20}\end{array}$ & $\begin{array}{l}35 \text { sujeitos,VEF } \\
\text { (\% predito): }<50 \%\end{array}$ & $\begin{array}{l}\text { Variáveis analisadas antes } \\
\text { da execução da RFL, } \\
\text { imediatamente após a manobra } \\
\text { e } 5 \text { minutos mais tarde. }\end{array}$ & $\begin{array}{r}\mathrm{Cl}, \mathrm{VIF}_{1}, \mathrm{CVFi}, \mathrm{FImáx}_{50 \%} \text {, } \\
\text { pico de fluxo } \\
\text { inspiratório, } \mathrm{CV}, \mathrm{VEF}_{1}, f \text {, } \\
\text { tensão final } \mathrm{CO}_{2} \mathrm{e} \mathrm{SpO}_{2}\end{array}$ & $\begin{array}{l}\text { Os resultados demonstraram melhora } \\
\text { da } \mathrm{Cl} \text { e } \mathrm{SpO}_{2} \mathrm{com} \text { a adoção da manobra } \\
\text { e diminuição da } f \text {, tensão final } \mathrm{CO}_{2} \text {. Após } \\
\text { cinco minutos da realização da RFL, os } \\
\text { efeitos diminuíram. }\end{array}$ \\
\hline
\end{tabular}

VEF : volume expiratório forçado no primeiro minuto; RE: respiração espontânea; RFL: respiração frenolabial; Vcw : Volume expiratório final da caixa torácica; VAB : Volume expiratório final do compartimento abdominal; CRF: capacidade residual funcional; $V_{C W}$ : volume inspiratório final da caixa torácica; VRC : Volume inspiratório final do compartimento costal; VCcW: volume corrente da caixa torácica; VRC : volume expiratório final do compartimento costal; VC: volume corrente: TI: tempo inspiratório; TE: tempo expiratório; T tempo total; AVD: atividade de vida diária; GOLD: Global Initiative for Chronic Obstructive Lung Disease; ESWT: endurance shuttle walk test; $\mathrm{SpO}_{2}$ : saturação parcial de oxigênio; FC: frequência cardíaca; VFC: variabilidade da frequência cardíaca; PA: pressão arterial; RMMSD: raiz quadrada da média do quadrado das diferenças entre intervalos RR normais adjacentes; $\Delta \mathrm{I}$-E: variação da frequência cardíaca inspiração/expiração; $\Delta$ R-RFL: variação da frequência cardíaca repouso/RFL; Wpeak: carga de trabalho; $\mathrm{VO}_{2}$ : consumo de oxigênio; $\mathrm{VE}$ : ventilação por minuto; $\mathrm{CVF}$ : capacidade vital forçada; $\mathrm{Cl}$ : capacidade inspiratória; $\mathrm{VIF}$; volume inspiratório forçado no primeiro segundo; CVFi: capacidade vital forçada inspiratória; Flmáx $50 \%$ : fluxo inspiratório máximo à $50 \%$ da capacidade vital; CV: capacidade vital; f: frequência respiratória; $\mathrm{CO}_{2}$ : dióxido de carbono

variabilidade dos escores do Borg, justificada pela redução da $f$ e do prolongamento do TE.

Em 2007, Bianchi et al. ${ }^{16}$ avaliaram os possíveis efeitos da RFL sobre a dispneia. Os resultados foram expressos por meio de dois grupos: o primeiro, euvolumes, apresentou com a RFL uma diminuição do volume expiratório final da caixa torácica e abdominal abaixo da capacidade residual funcional (CRF), e aumento dos volumes inspiratórios finais da caixa torácica e do gradil costal, o que contribuiu para aumento do VC da caixa torácica e decréscimo da dispneia. Já o segundo grupo, hiperinsuflados, obteve, por meio da RFL, valores mais baixos do $\mathrm{VC}$ da caixa torácica em relação ao primeiro grupo, aumento nos volumes expiratórios finais acima da CRF e elevação do volume inspiratório final de ambos os compartimentos da caixa torácica. Esse padrão não apresentou associação com mudanças na sensação de dispneia durante o protocolo. A contribuição abdominal para o aumento do VC marca a diferença entre os dois grupos, auxiliando na limitação do trabalho inspiratório e repercutindo de forma positiva na sensação de dispneia.

Além das investigações realizadas durante o repouso, Spahija et al. ${ }^{14}$ avaliaram os efeitos da manobra sobre 
a mecânica ventilatória, o volume expiratório final e a dispneia de pacientes com DPOC durante exercício físico em bicicleta ergométrica. Os pacientes apresentaram respostas variáveis quanto à dispneia apenas durante o exercício, sendo positiva quando houve redução no volume expiratório final com consequente repercussão na geração de força muscular inspiratória, proporcionando recrutamento muscular da caixa torácica e da musculatura acessória durante a inspiração e a atuação da musculatura abdominal expiratória, refletindo de forma direta na atuação diafragmática em gerar pressão durante a inflação do pulmão.

\section{Respiração frenolabial e padrão respiratório e capacidade funcional}

A influência da RFL sobre o padrão respiratório foi estudada tanto em repouso quanto em exercício. Em repouso, a manobra promoveu aumento do VC, TI, TE, $\mathrm{T}_{\text {Tот }}{ }^{12,16}$ e da relação volume corrente/tempo inspiratório, bem como diminuição da $f$ e da relação tempo inspiratório/tempo total ${ }^{12}$. Já em exercício, foram observados prolongamento do $\mathrm{T}_{\mathrm{TOT}}$, com aumento do $\mathrm{VC}$, diminuição da $f$ e volume minuto inalterado ${ }^{14}$.

Visser et al. ${ }^{20}$ avaliaram os efeitos da RFL sobre os parâmetros inspiratórios neste tipo de população. Os resultados demonstraram melhora na capacidade inspiratória $(\mathrm{CI})$ e aumento de saturação, diminuição de frequência cardíaca e tensão de $\mathrm{CO}$, analisados secundariamente. Os autores relataram que melhora na CI indica menor hiperinsuflação com a adoção da técnica e que esta não promoveu alterações em parâmetros de fluxo.

Além disso, Garrod et al. ${ }^{13}$, ao avaliarem a RFL em pacientes que não a adotavam espontaneamente, durante um teste incremental de caminhada, observaram menores valores de $f$ pós-teste e retorno mais rápido aos níveis basais de dispneia no tempo de recuperação do Incremental Shuttle Walking Test (ISWT), quando utilizada a RFL em comparação com a respiração espontânea. Não foram encontrados benefícios em relação à dispneia durante o esforço, justificado pelos autores pela forma subjetiva de sua classificação bem como pelo período curto entre os testes (20 minutos), que pode ter influenciado as respostas entre eles e sobre a distância percorrida. Os pacientes que apresentaram diferença maior que seis respirações por minuto (rpm), entre o teste realizado com RFL e com respiração espontânea, foram classificados como 'responders', os quais apresentaram maiores escores de dispneia em repouso, avaliados pela Escala de Borg, além de uma tendência a menores valores de $\mathrm{VEF}_{1}$ em relação ao predito e à distância basal percorrida.

De forma contrária, em relação à capacidade funcional, os achados de Faager et al. ${ }^{17}$ mostraram que pacientes que utilizaram a RFL de forma espontânea, durante a execução do Endurance Shuttle Walking Test (ESWT), aumentaram o tempo de execução do teste, em média, 37 segundos em comparação aos que realizaram a respiração espontânea, o que indica uma maior resistência à realização do teste. Além disso, houve uma queda menor da $\mathrm{SpO}_{2}$ durante o teste realizado com a técnica, o que indica melhora regional da ventilação considerada de relevância clinica pelos autores. Já Nield et al. ${ }^{15}$ observaram ganho tanto sobre a capacidade funcional quanto sobre a dispneia após um treinamento de 12 semanas com a RFL, justificados por possíveis mecanismos fisiológicos, tais como redução da hiperinsuflação dinâmica por meio do prolongamento da expiração e aumento da força muscular inspiratória. Os autores reforçam o benefício da técnica, considerando-a uma manobra simples de ser realizada em atividades diárias, sem limitações ou restrições de uso.

\section{Respiração frenolabial e consumo de oxigênio}

Jones et al. ${ }^{11}$, ao compararem os efeitos de três tipos de padrões respiratórios (RFL, respiração diafragmática e suas associações) e respiração espontânea em pacientes com DPOC sobre o consumo de oxigênio, por meio da mensuração dos gases expirados durante o repouso, observaram que alterações no padrão ventilatório resultam em menores custos metabólicos, com menores valores de $\mathrm{VO}_{2}$ para a RFL, o que foi associado à diminuição da frequência respiratória. Segundo os autores, nestes pacientes, o aumento da ventilação minuto para compensar o aumento da resistência das vias aéreas e da complacência pulmonar, devido à diminuição do recolhimento elástico do parênquima pulmonar, eleva o consumo de oxigênio e, consequentemente, o custo metabólico.

A interação da realização da RFL com o gasto energético foi também avaliada durante exercício em bicicleta ergométrica, por Spahija et al. ${ }^{19}$. Os autores observaram que os indivíduos que adotaram de forma espontânea a RFL durante o repouso - exercício - recuperação, bem como aqueles que executaram-na somente durante o exercício e recuperação, apresentaram valores inferiores do consumo de oxigênio. Os pacientes que adotaram a manobra durante todo o tempo eram os mais hipercapneicos, com menor tolerância ao exercício e capacidade 
de difusão e maior limitação do fluxo aéreo e hiperinflados. Segundo os autores, a severidade da obstrução e o grau de dispneia durante exercício desempenham papel importante para adoção ou não da RFL, de forma espontânea por pacientes com DPOC ${ }^{19}$.

Com base nos achados, pode-se afirmar que a adoção da RFL por pacientes com DPOC minimiza o consumo energético, o que contribui de maneira positiva sobre o padrão ventilatório desses indivíduos.

\section{Respiração frenolabial e modulação autonômica cardíaca}

Um único estudo publicado em 2009 abordou a influência da RFL sobre a modulação autonômica cardíaca. Ramos et al. ${ }^{18}$ estudaram a manobra realizada durante o repouso e observaram que sua realização eleva o valor do índice de raiz quadrada da média do quadrado das diferenças entre intervalos RR normais adjacentes (RMSSD), indicativo da atividade parassimpática, quando comparado à respiração espontânea. Os autores inferem que a RFL diminui o balanço simpatovagal, o que pode ser decorrente da diminuição da $f$. Em função das alterações observadas em parâmetros cardiorrespiratórios e autonômicos, os autores ressaltam a importância da monitorização destes pacientes durante a realização da manobra.

\section{Limitações do estudo}

A escassez de estudos que abordam a RFL como tema principal tornou-se uma limitação metodológica da busca realizada, pois impossibilitou uma seleção mais criteriosa em relação ao desenho do estudo dos artigos selecionados para compor esta revisão.

\section{CONCLUSÕES}

Os trabalhos demonstram que a RFL promove modificações benéficas em diversos sistemas, dentre eles o respiratório e o autonômico, além de benefícios na qualidade de vida, o que evidencia a importância de sua utilização em paciente com DPOC, tanto em programas de reabilitação quanto de forma espontânea em atividades de vida diária. Ressalta-se, ainda, a importância na orientação da técnica pelos profissionais que a utilizam, devido às modificações promovidas com sua realização.

\section{REFERÊNCIAS}

1. Barach AL. Physiologic advantages of grunting, groaning, and pursed-lip breathing: adaptive symptoms related to the development of continuous positive pressure breathing. Bull N Y Acad Med. 1973;49(8):666-73

2. Azeredo CAC, Fonseca MF. Padrões ventilatórios. In: Azeredo. Fisioterapia respiratória atual [s.l; s.n]. 1987;63-122.

3. Spahija JA, Grassino A. Effects of pursed-lips breathing and expiratory resistive loading in healthy subjects. J Appl Physiol. 1996;80: 1772-84.

4. Ugalde V, Breslin EH, Walsh SA, Bonekat HW, Abresch RT, Carter GT. Pursed lips breathing improves ventilation in myotonic muscular dystrophy. Arch Phys Med Rehabil. 2000;81(4):472-8.

5. Thoman RL, Stoker GL, Ross JC. The efficacy of pursed-lips breathing in patients with chronic obstructive pulmonary disease. Am Rev Respir Dis. 1966;93:100.

6. Mueller RE, Petty TL, Filley GF. Ventilation and arterial blood gas changes induced by pursed lips breathing. J Appl Physiol. 1970;28:784-9.

7. Tiep BL, Burns M, Kao D, Madison R, Herrera J. Pursed lips breathing training using ear oximetry. Chest. 1986;90:218-21.

8. Breslin EH. The pattern of respiratory muscle recruitment during pursed-lip breathing. Chest. 1992:101:75-8.

9. Roa J, Epstein S, Breslin E, Shannon T, Celli B. Work of breathing and ventilatory muscle recruitment during pursed lips breathing in patients with chronic airway obstruction [resumen]. Am Rev Respir Dis. 1991;143:A77.

10. Schmidt RW, Wasserman K, Lillington GA. The effect of airflow and oral pressure on the mechanics of breathing in patients with asthma and emphysema. Am Rev Respir Dis. 1964;90:564-71.

11. Jones AYM, Dean E, Chow CCS. Comparison of the oxygen cost of breathing exercises and spontaneous breathing in patients with stable chronic obstructive pulmonary disease. Phys Ther. 2003;83(5):424-31.

12. Bianchi R, Gigliotti F, Romagnoli I, Lanini B, Castellani C, Grazzini M, et al. Chest wall kinematics and breathlessness during pursed-lip breathing in patients with COPD. Chest. 2004;125(2):459-65.

13. Garrod R, Dallimore K, Cook J, Davies V, Quade K. An evaluation of the acute impact of pursed lips breathing on walking distance in nonspontaneous pursed lips breathing chronic obstructive pulmonary disease patients. Chron Respir Dis. 2005;2(2):67-72.

14. Spahija J, Marchie M, Grassino A. Effects of imposed pursed-lips breathing on respiratory mechanics and dyspnea at rest and during exercise in COPD. Chest. 2005;128(2):640-50.

15. Nield MA, Soo Hoo GW, Roper JM, Santiago S. Efficacy of pursedlips breathing: a breathing pattern retraining strategy for dyspnea reduction. J Cardiopulm Rehabil Prev. 2007;27(4):237-44.

16. Bianchi R, Gigliotti F, Romagnoli I, Lanini B, Castellani C, Binazzi B, et al. Patterns of chest wall kinematics during volitional pursed-lip breathing in COPD at rest. Respir Med. 2007:101(7):1412-8.

17. Faager G, Stahle A, Larsen FF. Influence of spontaneous pursed lips breathing on walking endurance and oxygen saturation in patients with moderate to severe chronic obstructive pulmonary disease. Clin Rehabil. 2008;22(8):675-83.

18. Ramos EMC, Vanderlei LCM, Ramos D, Teixeira LM, Pitta F, Veloso M. Influence of pursed-lip breathing on heart rate variability and 
cardiorespiratory parameters in subjects with chronic obstructive pulmonary disease (COPD). Rev Bras Fisioter. 2009;13(3):288-93.

19. Spahija J, Marchie M, Ghezzo H, Grassino H. Factors discriminating spontaneous pursed-lips breathing use in patients with COPD. COPD. 2010;7(4):254-61.

20. Visser FJ, Ramlal S, Dekhuijzen PN, Heijdra YF. Pursed-Lips Breathing Improves Inspiratory Capacity in Chronic Obstructive Pulmonary Disease. Respiration. 2011;81:372-8.
21. Fregonezi GA, Resqueti VR, Güell Rous R. Pursed lips breathing. Arch Bronconeumol. 2004:40(6):279-82.

22. Dechman G, Wilson CR. Evidence Underlying Breathing Retraining in People With Stable Chronic Obstructive Pulmonary Disease. Phys Ther. 2004;84(12):1189- 2004

23. Dourado VZ, Tanni SE, Vale SA, Faganello MM, Sanchez FF, Godoy I. Manifestações sistêmicas na doença pulmonar obstrutiva crônica. J Bras Pneumol. 2006;32(2):161-71. 